\title{
Custom JPEG Quantization for Improved Iris Recognition Accuracy
}

\author{
Gerald Stefan Kostmajer ${ }^{1}$, Herbert Stögner ${ }^{1}$, and Andreas Uhl ${ }^{2}$ \\ 1 School of Communication Engineering for IT, Carinthia Tech Institute, Austria \\ 2 Department of Computer Sciences, University of Salzburg, Austria \\ uhl@ecosy.sbg.ac.at
}

\begin{abstract}
Custom JPEG quantization matrices are proposed to be used in the context of compression within iris recognition. Superior matching results in terms of average Hamming distance and improved ROC is found as compared to the use of the default quantization table especially for low FAR. This leads to improved user convenience in case high security is required.
\end{abstract}

\section{Introduction}

With the increasing usage of biometric systems the question arises naturally how to store and handle the acquired sensor data. In this context, the compression of these data may become imperative under certain circumstances due to the large amounts of data involved. Among other possibilities (e.g. like template storage on IC cards), compression technology may be used in two stages of the processing chain in classical biometric recognition:

1. Transmission of sample data after sensor data acquisition: In distributed biometric systems, the data acquisition stage is often dislocated from the feature extraction and matching stage (this is true for the enrollment phase as well as for authentication). In such environments the sensor data have to be transferred via a network link to the respective location, often over wireless channels with low bandwidth and high latency. Therefore, a minimization of the amount of data to be transferred is highly desirable, which is achieved by compressing the data before transmission. An alternative solution would be to extract the features before transmission and to transfer feature data only - in many cases, feature extraction is more demanding as compared to compression which generates additional workload for the often mobile and low power acquisition devices.

2. Storage of reference data: In most template databases (where the reference data of the enrolled individuals is stored) only the extracted features required for the matching step are stored as opposed to retaining the originally acquired sensor data. However, in case the features should be replaced for some reason (e.g. when a superior or license-free matching technique involving a different feature set becomes available), having stored only extracted features implies the requirement for all legitimate users for a re-enrollment, which can be expensive and is highly undesired since user-acceptance of the entire biometric system will suffer. Storing the original

D. Gritzalis and J. Lopez (Eds.): SEC 2009, IFIP AICT 297, pp. 76-86, 2009.

(c) IFIP International Federation for Information Processing 2009 
sensor data in addition to the features required for the current matching technique solves this problem. Of course, these data need to be stored in compressed (to save storage space) and encrypted (to protect privacy) form.

Having found that compression of the raw sensor data can be advantageous in certain applications, we have to identify techniques suited to accomplish this task in an optimal manner. In order to maximize the benefit in terms of data reduction, lossy compression techniques have to be applied. However, the distortions introduced by compression artifacts usually interfere with subsequent feature extraction and may degrade the matching results. In particular, FRR or FNMR will increase (since features of the data of legitimate users are extracted less accurately from compressed data) which in turn affects user convenience and general acceptance of the biometric system. In extreme cases, even FAR or FMR might be affected.

In this work, we will focus on the lossy compression of iris images using the JPEG standard. We discuss the use of custom quantization matrices in order to reflect the specific properties of iris imagery. Contrasting to the overwhelming majority of literature and studies in the field of compressing biometric sample data, we will not rely on assessing the resulting objective and subjective image quality after compression, but we will apply a biometric iris recognition systems to the compressed sensor data to evaluate the effects of compression on recognition accuracy, in particular on the matching results of legitimate and illegitimate users.

In Section 2, we will review and discuss the available literature on biometric sample data compression with focus on iris data storage. Section 3 is the main part of this work where we discuss properties of iris imagery and present several variants of custom JPEG quantization matrices (designed in order to hopefully improve recognition accuracy). In section 4 we first describe the employed iris recognition system and the data this algorithm are applied to. Subsequently we discuss our experimental results with respect to the observed improvements of recognition accuracy.

\section{Iris Image Compression}

Iris recognition is claimed to be the most secure biometric modality exhibiting practically $0 \%$ FAR and low FRR. An interesting fact is that the iris recognition market is strongly dominated by Iridian Inc. based technology which is based on algorithms of J. Daugman [2]. The certainly most relevant standard for compressing iris image data is the recent ISO/IEC 19794-6 standard on Biometric Data Interchange Formats.

While the data formats specified by the ISO/IEC 19794 standard are fixed at present state, their customized use tailored to a specific target modality and the corresponding impact on recognition accuracy as compared to the default settings has not been investigated. This is the scope of the current paper.

ISO/IEC 19794-6 allows iris image data to be stored in lossy manner in the JPEG and JPEG2000 formats. Two types of iris image data are considered: rectilinear images (i.e. images of the entire eye) and polar images (which are basically the result of iris detection and segmentation), the latter much smaller in terms of storage requirement (e.g. $2 \mathrm{kB}$ vs. $25-30 \mathrm{kB}$ for rectilinear images). It is important to note that with this standardization it 
might become more attractive for companies to investigate alternatives to Iridian products due to the available common data format iris recognition systems can rely on.

Only recently, first results and techniques are available on iris image compression and its impact on recognition performance. Ives et al. [5]6] apply JPEG2000 up to a compression rate of 20 to rectilinear image data (the CASIA database and a proprietary image collection is used) and investigate the effects on FAR and FRR of a 1-D version of the Daugman algorithm (the same system which is used in this study). Rakshit and Monro [11] again use JPEG2000 to compress polar iris images up to a compression rate of 80 and study the impact on verification accuracy of three iris recognition systems (including the Daugman algorithm, the CASIA database is used). Daugman and Downing [3] apply JPEG and JPEG2000 to rectilinear image data (the NIST ICE database is used) and remove image background (i.e. parts of the image not being part of the eye like eye-lids are replaced by constant average gray) before compression is applied. A more compact way of representing the Daugman IrisCode is discussed in [12], however, these results refer to template compression and are only valid for the techniques related to Iridian products. In previous work [97], we have compared five general purpose compression algorithms (including JPEG and JPEG2000) with respect to their impact on iris recognition accuracy of three different recognition schemes (the CASIA database has been used). In accordance to [3] superior compression performance of JPEG2000 over JPEG is found especially for low bitrates, however, for high and medium quality JPEG is still an option to consider. So far, compression algorithms have been applied to iris imagery with their respective standard settings.

In the subsequent study we apply JPEG as covered by ISO/IEC 19794-6 to rectilinear iris images and propose to use custom quantization matrices adapted to properties of iris imagery. Contrasting to the optimization of the JPEG quantization matrix with respect to human perception as done for the development of the standard matrix, rate/distortion criteria have also been used successfully for the design of this matrix (see e.g. [4]). In [1] compression algorithms tuned for application in the pattern recognition context are proposed, which are based on the modification of the standard compression algorithms: this is done by emphasizing middle and high frequencies and discarding low frequencies (the standard JPEG quantization matrix is rotated by 180 degrees). JPEG quantization matrix optimization has already been considered in biometrics - [8] employ a rate/distortion criterion in the context of face recognition and achieve superior recognition performance as compared to the standard matrix.

\section{Custom JPEG Quantization}

The JPEG still image compression standard [10] allows to use custom quantization tables (Q-tables) in case image material with special properties is subject to compression. These tables are signalled in the header information. The default quantization matrices have been designed with respect to psychovisual optimality employing large scale experimentation involving a high number of test subjects. There are two reasons which suggest to use different Q-tables as the default configuration: First, iris imagery might have different properties as compared to common arbitrary images, and second, a pleasant viewing experience as being the aim in designing the default tables, might 
not deliver optimal matching results in the context of biometric recognition (e.g. sharp edges required for exact matching could appear appealing to human observers).

Therefore, as a first stage, we have investigated iris imagery in more detail. 8x8 pixel blocks have been subjected to DCT transform and the resulting coefficients are averaged for a large number of blocks (i.e. 2000, 525, and 44160 blocks for the three types of imagery, respectively). As a first class of blocks, we have used arbitrary images and blocks are extracted randomly. The second class of blocks is extracted iris texture taken left and right of the pupil while the third class is taken from polar iris images generated by the employed matching algorithm (see below). Fig. 1 displays the result of all three classes where the DC and the largest AC coefficient are set to white, zero is set to black and the remaining values are scaled in between (note that the logarithm is applied to the magnitude of all coefficients before this scaling operation).

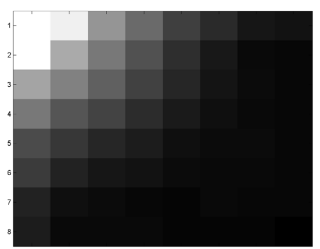

(a) arbitrary blocks

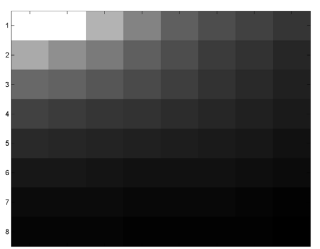

(b) polar iris blocks

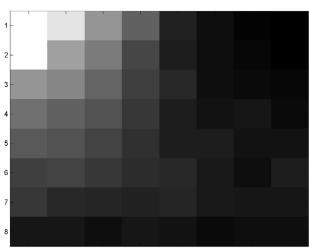

(c) horizontal iris blocks

Fig. 1. Averaged 8x8 DCT blocks

The arbitrary blocks (Fig. 1,a) show the typical expected behaviour with decreasing coefficient magnitude for increasing frequency and symmetry with respect to the coordinate axes. Fig. 1 b reveals that in polar iris images there is more energy in the higher frequencies in horizontal direction as compared to vertical direction. This is to be expected since luminance fluctuations in iris texture are more pronounced in radial direction as compared to perpendicular direction. Finally, Fig. 1. confirms this expectation showing more energy in the higher frequencies in vertical direction.

While we cannot exploit the direction bias of iris texture in compression since we are dealing with rectangular iris images, we conjecture that the highest and medium

\begin{tabular}{|l|l|l|l|l|l|l|l|}
\hline 16 & 11 & 10 & 16 & 24 & 40 & 255 & 255 \\
\hline 12 & 12 & 14 & 19 & 26 & 255 & 255 & 255 \\
\hline 14 & 13 & 16 & 24 & 255 & 255 & 255 & 255 \\
\hline 14 & 17 & 22 & 255 & 255 & 255 & 255 & 255 \\
\hline 18 & 22 & 255 & 255 & 255 & 255 & 255 & 255 \\
\hline 24 & 255 & 255 & 255 & 255 & 255 & 255 & 255 \\
\hline 255 & 255 & 255 & 255 & 255 & 255 & 255 & 255 \\
\hline 255 & 255 & 255 & 255 & 255 & 255 & 255 & 255 \\
\hline
\end{tabular}

(a) Q-table 12

\begin{tabular}{|l|l|l|l|l|l|l|l|}
\hline 16 & 11 & 10 & 16 & 24 & 255 & 255 & 255 \\
\hline 12 & 12 & 14 & 19 & 255 & 255 & 255 & 255 \\
\hline 14 & 13 & 16 & 255 & 255 & 255 & 255 & 255 \\
\hline 14 & 17 & 255 & 255 & 255 & 255 & 255 & 255 \\
\hline 18 & 255 & 255 & 255 & 255 & 255 & 255 & 255 \\
\hline 255 & 255 & 255 & 255 & 255 & 255 & 255 & 255 \\
\hline 255 & 255 & 255 & 255 & 255 & 255 & 255 & 255 \\
\hline 255 & 255 & 255 & 255 & 255 & 255 & 255 & 255 \\
\hline
\end{tabular}

(b) Q-table 13

\begin{tabular}{|l|l|l|l|l|l|l|l|}
\hline 16 & 11 & 10 & 16 & 255 & 255 & 255 & 255 \\
\hline 12 & 12 & 14 & 255 & 255 & 255 & 255 & 255 \\
\hline 14 & 13 & 255 & 255 & 255 & 255 & 255 & 255 \\
\hline 14 & 255 & 255 & 255 & 255 & 255 & 255 & 255 \\
\hline 255 & 255 & 255 & 255 & 255 & 255 & 255 & 255 \\
\hline 255 & 255 & 255 & 255 & 255 & 255 & 255 & 255 \\
\hline 255 & 255 & 255 & 255 & 255 & 255 & 255 & 255 \\
\hline 255 & 255 & 255 & 255 & 255 & 255 & 255 & 255 \\
\hline
\end{tabular}

(c) Q-table 15

\begin{tabular}{|r|r|r|r|l|l|l|l|}
\hline 16 & 11 & 10 & 255 & 255 & 255 & 255 & 255 \\
\hline 12 & 12 & 255 & 255 & 255 & 255 & 255 & 255 \\
\hline 14 & 255 & 255 & 255 & 255 & 255 & 255 & 255 \\
\hline 255 & 255 & 255 & 255 & 255 & 255 & 255 & 255 \\
\hline 255 & 255 & 255 & 255 & 255 & 255 & 255 & 255 \\
\hline 255 & 255 & 255 & 255 & 255 & 255 & 255 & 255 \\
\hline 255 & 255 & 255 & 255 & 255 & 255 & 255 & 255 \\
\hline 255 & 255 & 255 & 255 & 255 & 255 & 255 & 255 \\
\hline
\end{tabular}

(d) Q-table 16

Fig. 2. JPEG Quantization tables 
frequencies might not be required for he matching stage due to the coarse quantization used for template generation while at least medium frequencies are required for pleasant viewing. Fig. 2 displays the Q-tables used in our experiments.

From left to right, an increasing amount of high frequencies is suppressed following the zig-zag scan known from JPEG bitstream generation (by dividing the coefficients by 255), coefficients not affected are quantized as defined in the default Q-table. For the rightmost Q-table 16, only the 6 leading coefficients are quantized in the regular manner, the rest is severely quantized. The rationale behind the selection of these matrices is to investigate the importance of medium frequency information in iris recognition (high frequency information is assumed to be not useful in any case).

\section{Experimental Study}

\subsection{Setting and Methods}

\subsubsection{Iris Recognition System}

The employed iris recognition system is Libor Masek's Matlab implementation 11 of a 1-D version of the Daugman iris recognition algorithm. First, this algorithm segments the eye image into the iris and the remainder of the image. Iris image texture is mapped to polar coordinates resulting in a rectangular patch which is denoted "polar image". After extracting the features if the iris (which are strongly quantized phase responses of complex 1-D Gabor filters in this case), considering translation, rotations and disturbed regions in the iris (a noise mask is generated), the algorithm outputs the similarity score by giving the hamming distance between two extracted templates. The range of the hamming distance reaches from zero (ideal matching of two iris images of the same person) to 0.5 (ideal mismatch between two iris images of different persons).

\subsubsection{Sample Data}

For all our experiments we considered 320x280 pixel images with 8-bit grayscale information per pixel from the CASIA 1.0 iris image database. For rectilinear iris images, we applied the experimental calculations on the images of 100 persons using 3 images for each eye (i.e. 600 images). Note that it makes an important difference if compression is applied to rectangular or polar iris images which has an important implication on the performance of the entire system. Whereas in the case of compressing polar iris images [11] only the iris texture information is affected, in the case of compressing rectangular image data also the iris detection and determination of the noise mask is potentially affected in addition to degrading texture information. Figure 3 shows an example of a JPEG2000 compressed (compression rate 96) iris image of one person, together with the extracted iris template data and the noise masks (template and noise mask have been scaled in y-direction by a factor of 4 for proper display). The noise mask is hardly affected by the compression, whereas in the two templates differences resulting from compression artifacts are clearly observable.

\footnotetext{
1 http://www.csse.uwa.edu.au/ pk/studentprojects/libor/ sourcecode.html

2 http://www.sinobiometrics.com
} 

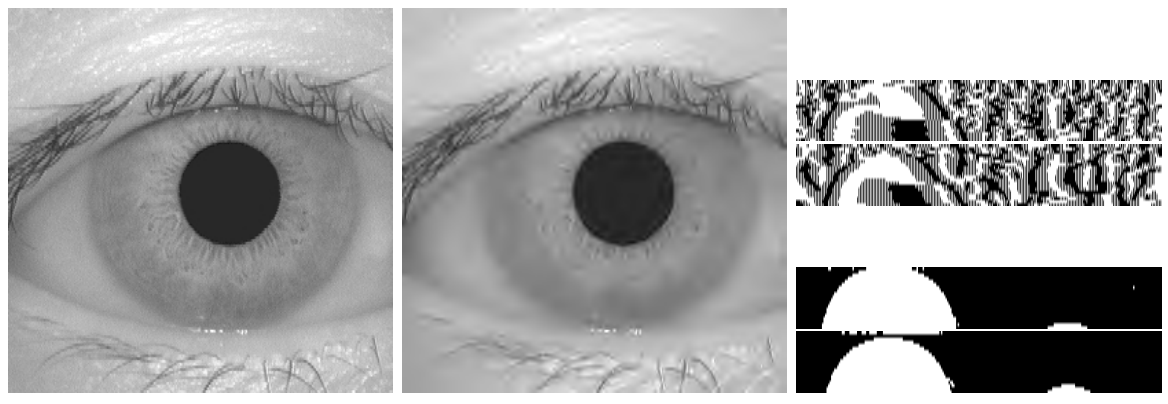

Fig. 3. Comparison of uncompressed/compressed iris image and the corresponding iris templates and noise masks

Compression can be used in various stages of the matching process. Either the stored reference data may be in compressed form, or the sample data acquired for verification may be compressed (e.g. for efficient transfer), or both. Therefore, we use two settings in our experiments: either both images are compressed and matched against each other or only one image is compressed in the matching stage. For investigating correct matches (matches from legitimate users enrolled in the database), we rely on 12000 generated images (i.e. for each of the 100 persons, we have 3 images for each eye resulting in 3 ! possible correct matches for each eye; for 200 eyes, this totals in 1200 images per compression rate; considering the 10 different compression rates we finally result in 12000 overall images considered). This is only true in the scenario with only 1 compressed image, for 2 compressed images this number is half-ed due to symmetry reasons. For investigating matches between different persons (imposter matches), far more data is available of course.

\subsection{Experimental Results}

Figure 4 a shows the averaged rate distortion comparison of the different compression algorithms applied to all iris images considered for three Q-tables. It is clearly displayed that employment of the default Q-table results in the best PSNR across the entire range of bitrates considered. Therefore, a corresponding matching behaviour (best results for the default Q-table) could be expected in the context of iris recognition.

In the following, we investigate the impact of compression on the matching score (i.e. obtained hamming distance (HD)). The interval of $0.26 \leq H D \leq 0.35$ is discussed as the border between match and mismatch in iris recognition [2] - based on recommendations for the specific technique [5] used and results shown subsequently we suggest to choose $H D=0.34$ as decision criterion between match and mismatch.

Fig. 4. b shows the plot of the HD after applying the iris recognition algorithm to both JPEG compressed iris images in the case of imposter matches (i.e. irises of different persons / eyes are matched against each other). The $\mathrm{x}$-axis shows the compression rates, whereas the $y$-axis shows the averaged hamming distance. For reference, we have included the average HD for the case of uncompressed images as horizontal dotted line in light gray (labelled UC). 


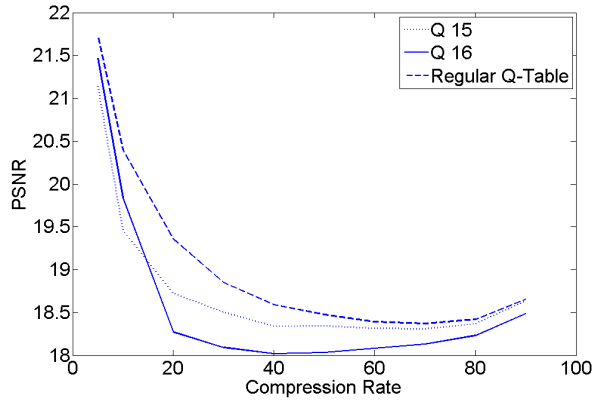

(a) PSNR

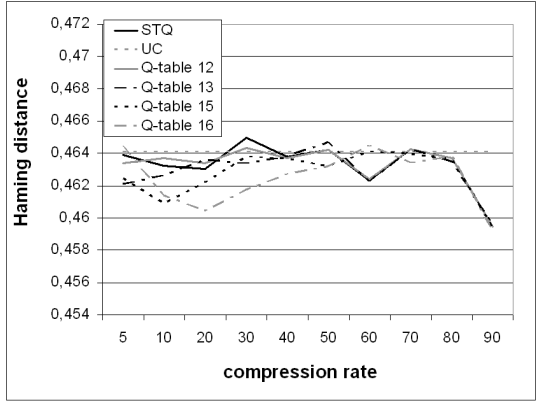

(b) HD (imposters)

Fig. 4. Impact of varying compression rate

For the case of imposters the HD remains above 0.46 across the whole range of compression rates for all sensible compression rates. This means that JPEG compression does not introduce any false positive matches on average no matter how severe compression is applied. Of course, this does not exclude the possibility of the existence of statistical outliers of course. There are no significant differences among the different Qtables since the fluctuations occur in a negligible range. The same behaviour is observed in case only one image is compressed (not shown).

In the case of genuine users (see Fig. 5), the mean value of the HD in the uncompressed case is approximately 0.31. First we consider the standard Q-table (labelled STQ). For increasing compression rate the HD stays constant at approximately 0.305 until the compression rate exceeds 10 and increases subsequently. A further increase of the compression rate leads to a steady increase of HD and crosses the suggested matching threshold of 0.34 between compression rates 30 and 40 . Note that the reported numbers refer to averaged HD values which implies the occurrence of a significant number of false negative matches at this compression rate. In the case of both images being compressed, HD is lower on average up to a compression rate of 20.

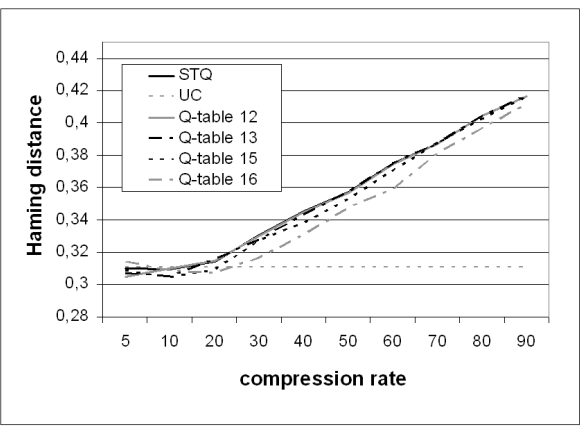

(a) one image compressed

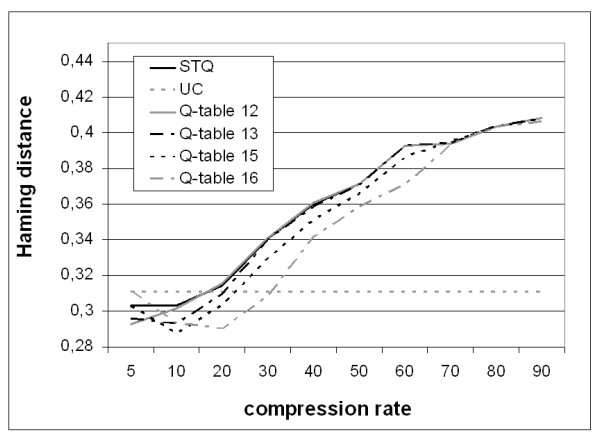

(b) both images compressed

Fig. 5. Impact of varying compression rate on HD of genuine users' matches 
When comparing these results to those obtained with different Q-tables, we notice that Q-tables 15 and 16 clearly improve on the results of STQ from compression rate 20 upwards where Q-table 16 does so in a more pronounced manner. For compression rate 10 all other Q-tables improve slightly on STQ and for compression rate 5, Q-tables 12 and 13 are superior to STQ in terms of average HD. In the case of two images being compressed, the observed behaviour is more significant but similar in principle.

These results indicate that PSNR is indeed NOT a good predictor for matching performance with compressed iris images in terms of average Hamming distance. The claim that compression up to a rate of 16 even improves the matching scores of not compressed images [11] can be supported at least for the 2 compressed images case and the STQ, for "better" Q-tables this is correct even up to compression rate 20 and higher.

In order to consider the hidden statistical outliers in the comparisons and to use a quantity often employed in the assessment of biometric system performance, we will focus on the receiver operating characteristic (ROC) by computing and plotting the false rejection rate (FRR) against the false acceptance rate (FAR) for different compression rates:

$$
\begin{gathered}
F R R=\frac{\text { Number of (false) negative matches }}{\text { Number of legitimate users' matches }} . \\
F A R=\frac{\text { Number of (false) positive matches }}{\text { Number of imposter matches }} .
\end{gathered}
$$

Figs. 6 to 8 compare the ROC of different Q-tables for compression rates 5, 10, and 20 since it is not realistic to operate the iris recognition system at a higher compression rate. Again, the two compressed image scenario is compared to the case where only one image is compressed.

For compression rate 5, our proposed Q-tables are not really able to substantially improve ROC. While for one compressed image (Fig. 6.b) only Q-table 15 improves STQ slightly (and only starting from $F R R>0.08$ ), significant improvements are seen for Q-tables 15, 16, and 13 (Fig. 6.a). However, only Q-table 15 starts improving at a reasonable low $F R R>0.04$.

In the case of compression rate 10, the situation changes drastically. Again, Q-table 15 shows the most significant improvements. For two compressed images (Fig. (7)a), at FAR 0.028, Q-table delivers a FRR of almost 0 whereas STQ exhibits an FRR of 0.15. Also the other proposed Q-tables improve on STQ in the interesting lower FRR range.

In the case of only one compressed image we still find improvements, but far less pronounced (Fig. (7) b): For an FAR of 0.041, again Q-table 15 gives FRR almost 0 whereas STQ is almost at FRR 0.9. Again, also Q-tables 13 and 16 improve on STQ.

Finally, when turning to compression rate 20 the situation is different (Fig. 8): now Q-table 16 shows the most significant improvements in the two compressed images case and shows behaviour similar to Q-table 15 also for one compressed image. The most noticeable improvement is found in the latter case at a FAR of 0.035 where Q-table 15 exhibits FRR close to 0 and STQ has an FRR of 1.2.

There is one more interesting thing to note: at least for compression rate 20 it is entirely clear that it is NOT advantageous to compress both images involved in matching- 


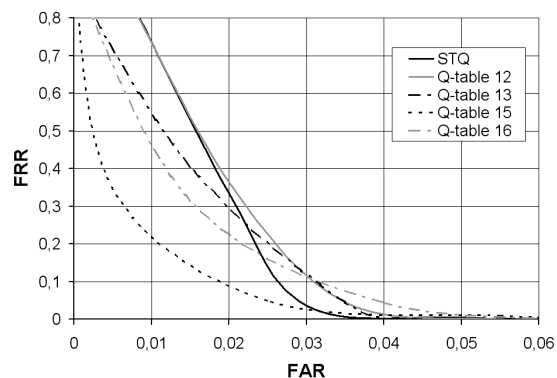

(a) 2 compressed images

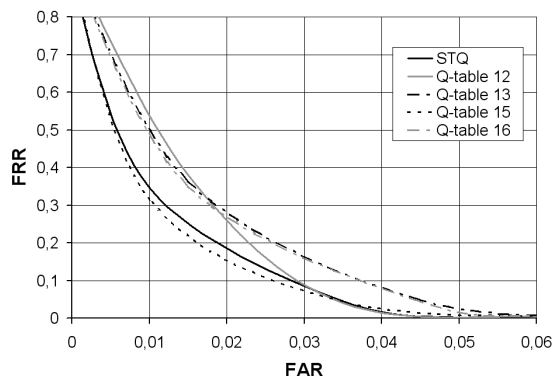

(b) 1 compressed image

Fig. 6. Compression rate 5

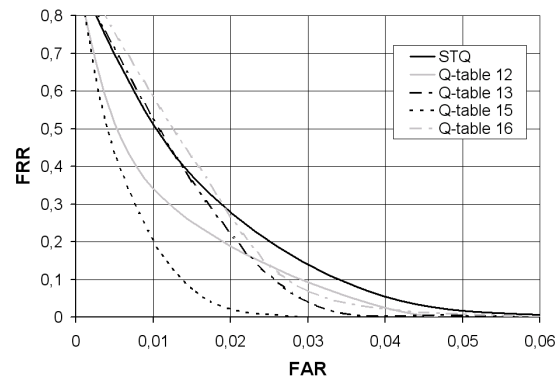

(a) 2 compressed images

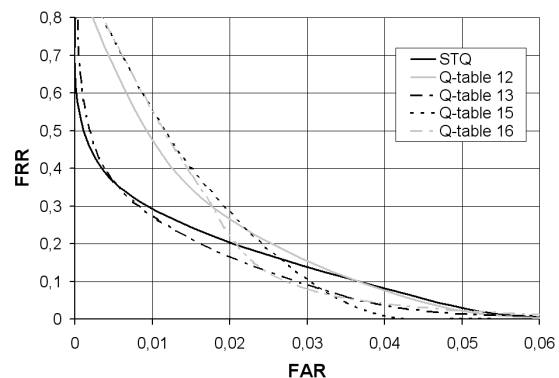

(b) 1 compressed image

Fig. 7. Compression rate 10

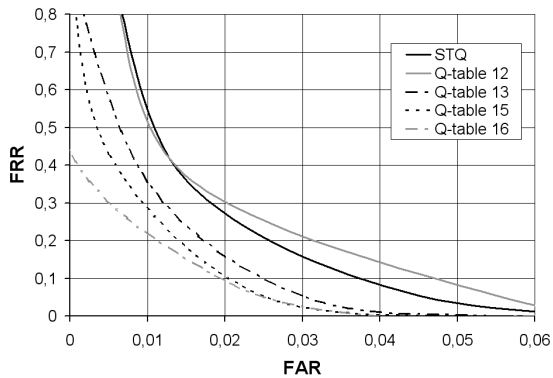

(a) 2 compressed images

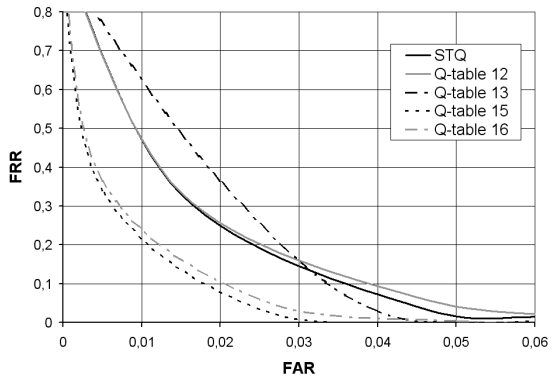

(b) 1 compressed image

Fig. 8. Compression rate 20

in terms of ROC, clearly the case of one compressed image is superior here. For compression rates 5 and 10 the better choice highly depends on the target FAR/FRR and the Q-table in use. 


\section{Conclusion and Future Work}

We have found that custom designed quantization tables in JPEG can improve matching results in terms of average HD and ROC behaviour. This is especially true for compression rates of 10 and higher where improvements are seen especially for low FAR. In this case, FRR can be limited much more effective as compared to the default quantization table. In addition to that we have found PSNR to be not at all suited to predict the recognition performance in iris recognition systems. The advantage of compressing both images involved in the matching process cannot be confirmed, in contrary, evidence for the opposite is found for higher compression rates.

In future work we will consider additional alternative iris recognition algorithms in order to identify possible interference between compression technique and iris recognition system. Furthermore we will employ optimization techniques (e.g. GAs) in order to design even more customized quantization tables for this application scenario.

\section{Acknowledgements}

Most of the work described in this paper has been done in the scope of the ILV "Compression Technologies and Data Formats" (winter term 2007/2008) in the master program on "Communication Engineering for IT" at Carinthia Tech Institute. The artificial name Gerald Stefan Kostmajer represents the following group of students working on this project: Eigner Raimund, Fischelschweiger Erik, Gernig Stefan, Jereb Martin, Kampusch Andreas, Konrad Mario, Lenzhofer Michael, Mataln Martin, Rabl Manuel, Schlögl Daniel, and Theuermann Klaus. This work has been partially supported by the Austrian Science Fund, project no. L554-N15.

\section{References}

1. Chen, M., Zhang, S., Karim, M.: Modification of standard image compression methods for correlation-based pattern recognition. Optical Engineering 43(8), 1723-1730 (2004)

2. Daugman, J.: How iris recognition works. IEEE Transactions on Circiuts and Systems for Video Technology 14(1), 21-30 (2004)

3. Daugman, J., Downing, C.: Effect of severe image compression on iris recognition performance. IEEE Transactions on Information Forensics and Security 3(1), 52-61 (2008)

4. Fong, W., Chan, S., Ho, K.: Designing JPEG quantization matrix using rate-distortion approachand human visual system model. In: Proceedings of the IEEE International Conference on Communications (ICC 1997), vol. 3, pp. 1659-1663 (1997)

5. Ives, R., Bonney, B., Etter, D.: Effect of image compression on iris recognition. In: IMTC 2005 - Instrumentation and Measurement Technology Conference (2005)

6. Ives, R.W., Broussard, R.P., Kennell, L.R., Soldan, D.L.: Effects of image compression on iris recognition system performance. Journal of Electronic Imaging 17, 011, 015 (2008), doi:10.1117/1.2891

7. Jenisch, S., Lukesch, S., Uhl, A.: Comparison of compression algorithms' impact on iris recognition accuracy II: revisiting JPEG. In: Proceedings of SPIE, Security, Forensics, Steganography, and Watermarking of Multimedia Contents X, vol. 6819, p. 68190M ff, San Jose, CA, USA (2008) 
8. Jeong, G.M., Kim, C., Ahn, H.S., Ahn, B.J.: JPEG quantization table design for face images and its application to face recognition. IEICE Transactions on Fundamentals of Electronics, Communications and Computer Science E69-A(11), 2990-2993 (2006)

9. Matschitsch, S., Tschinder, M., Uhl, A.: Comparison of compression algorithms' impact on iris recognition accuracy. In: Lee, S.-W., Li, S.Z. (eds.) ICB 2007. LNCS, vol. 4642, pp. 232-241. Springer, Heidelberg (2007)

10. Pennebaker, W., Mitchell, J.: JPEG - Still image compression standard. Van Nostrand Reinhold, New York (1993)

11. Rakshit, S., Monro, D.: Effects of sampling and compression on human iris verification. In: Proceedings of the IEEE International Conference on Acustics, Speech, and Signal Processing (ICASSP 2006), Tolouse, France, pp. II-337-II-340 (2006)

12. von Seelen, U.: IrisCode template compression and its effects on authentication performance. In: Biometrics Consortium Conference 2003 (2003) 